\title{
Role of CGF (Concentrated Growth Factor) in periodontal regeneration
}

\begin{abstract}
CGF-"A miracle in regenerative dentistry". CGF- Concentrated Growth Factor is a new regeneration platelet aggregate which is used widely in periodontal and oral surgeries. It is adopted without the use of chemicals which grades it more eco-friendly. It contains various growth factors which enhances its action and promotes wound healing. CGF is currently used along with autologous bone particles to induce bone regeneration and connective tissue attachment that shows excellent results .In future research can prove, that CGF can be used as a sole regenerative material. It is an excellent biomaterial which showcases back to normal periodontium with external finishing. This article focuses on the preparation, applications and advantages of using CGF in the field of regeneration.
\end{abstract}

Keywords: Concentrated growth factor, Periodontal regenerative procedures, Platelets growth factors
Volume 9 Issue 5 - 2018

\section{Nityasri, Aromal S, Pradeep KumarY, Kalaivani V, Raja Pandian \\ Department of Periodontology, SRM Kattankulathur Dental College, India}

Correspondence: Pradeep Kumar Y, Department of Periodontology, SRM Kattankulathur Dental College, Chennai, India, Email yadalam 1980@gmail.com

Received: September 04, 2018 | Published: September II, 2018
Abbreviations: CGF, Concentrated Growth Factor; PRP, Platelet-Rich Plasma; PRF, Platelet-Rich Fibrin; CAF, Coronally Advanced Flap; VEGF, Vascular Endothelial Growth Factor; EGF, Epidermal Growth Factor

\section{Introduction}

In dentistry, recent research of growth factors recognized that the best tissue regenerative stimulus is present amongst the autologous growth factors, which have clinically proven to induce regeneration and tissue healing. Growth factors are bioactive proteins which regulate a variety of cellular processes. Concentrated Growth Factors (CGF) was first developed by Sacco (2006), ${ }^{1}$ is a relatively new technology within the area of regenerative medicine. (CGF) is an advanced second generation platelet concentrate, obtained with differential continuous centrifugal technology, containing many kinds of growth factors and fibrins, and able to facilitate the recovery of soft and hard tissues.

CGF is different from platelet-rich plasma (PRP) and platelet-rich fibrin (PRF) in the methods for production because no additives are added during its production. CGF has a higher adhesive strength, tensile strength, higher viscosity than the other platelet preparations. CGF has a difference in centrifugation speed which permits the isolation of much larger and denser fibrin matrix richer in growth factors. CGF is a fibrin rich organic matrix which contains growth factors, platelets, leukocytes and CD34+ stem cells which help in the process of regeneration and also has immunological cells that are effective in regulating inflammation and minimizing the risk of infection. (CGF) is a biological inducing material which improves the quality of the formed bone, and facilitates the formation of bones and the healing of tissues.

\section{Preparation}

CGF is an autologous preparation; about $9 \mathrm{ml}$ of patient's venous blood is collected without anticoagulant solutions in sterile Vacuette tubes. The tubes are kept for centrifugation (Medifuge, Silfradent, Sofia, Italy) with one step centrifugation protocol: $30 \mathrm{sec}$-acceleration,
2 min - 2700 rpm, 4min 2400 rpm, 4min - 2700 rpm, 3 min - 3000 rpm, $36 \mathrm{sec}-$ deceleration and stop. This results in 4 different phases. ${ }^{1}$

\section{Phases of CGF}

1. Superior phase - Serum

2. Interim phase - Fibrin buffy coat

3. Liquid phase - Growth factors

4. Lower phase - Red blood cells

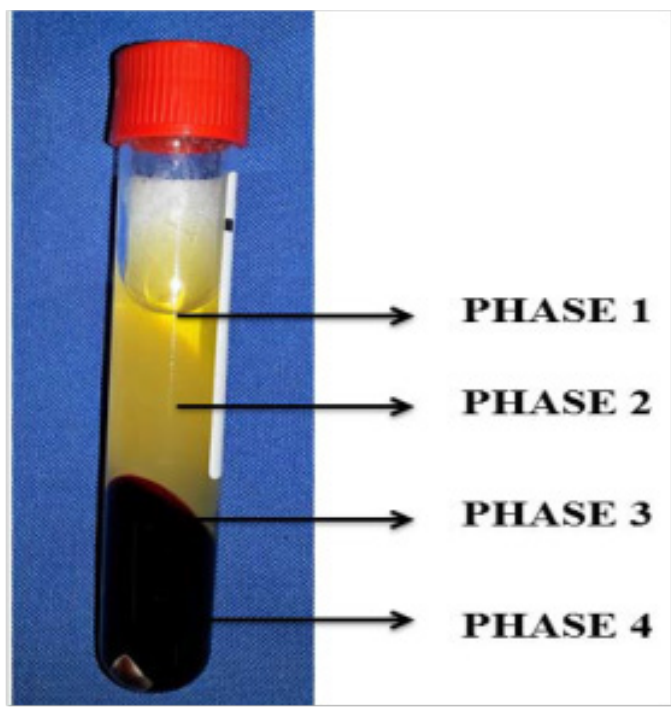

The CGF glue which is rich in growth factors is taken from the test tubes with tweezers and the two phases were cut off with scissors where the center and bottom layers connected. When the CGF glue is separated out, a quantity of growth factors are located on the interface between the CGF glue layer and the erythrocyte layer. Therefore, a certain amount of erythrocytes has to be retained when doing the separation to ensure the content of the growth factors. The CGF glue is pressed in moulds, squeezing the liquid elements within it and 
obtaining the CGF membrane. The CGF glue and the CGF membrane are put into sterile normal saline for future use. ${ }^{2}$

\section{Functions}

CGF is a fibrin tissue adhesive with haemostatic and tissue sealing properties. It promotes wound healing and accelerates osteogenesis. CGF improves the stability of the wound that is required for the attachment of a new connective tissue to the root surface. It promotes epithelial, endothelial and epidermal regeneration and decreases scarring. It has antimicrobial properties due to high concentration of leukocytes. It acts as an anti-antigenic agent on chronic non healing wounds. It also provides a scaffold supporting cytokine attachment and cellular migration.

\section{Applications}

\section{CGF in treatment of gingival recession}

CGF act as a membrane support in recession coverage as it constantly releases growth factors to produce tissue regeneration. According to the study ${ }^{3}$ it proved that CGF and CAF placed together enhance the healing of soft tissues. CGF is used as a barrier membrane to facilitate tissue healing and results in obtaining the attached gingival width in root coverage procedures like sliding flap technique.

According to this procedure, ${ }^{4}$ about $9 \mathrm{ml}$ of patient's venous blood is collected without anticoagulant solution and kept for centrifugation. After centrifugation for about twelve minutes the CGF was obtained and separated from the RBC layer. It is then placed in between the compressing disc to obtain a CGF membrane. The deepithelialisation was done around the root surface. The CGF membrane which was prepared is kept on the denuded root surface which acts as a barrier membrane, on top of which the lateral pedicle flaps are positioned. Sutures are placed covering the CGF membrane followed by dressing.

The sliding flap technique is significant as this flap has sufficient blood supply for a better healing. CGF membrane provides dual coverage to the exposed root surface. The membrane was exposed in few areas without covering the entire root surface. There were no complications during healing in unexposed areas, with the increase in width of attached gingiva.

\section{CGF in treatment of bone defects}

(CGF) is an excellent bioactive protein which enhances bone healing due to its stimulatory effect on epithelialization and angiogenesis. CGF is mixed with autologous bone particles or biomaterials to fill the bone defects to induce bone regeneration. The advantages of CGF over platelet-rich plasma are lack of biochemical modification, easy method of preparation, application with minimal expense. It also serves as a resorbable interpositional membrane. Avoids the early invigilation of the gingival epithelium is inhibited by the CGF layer results as a barrier to epithelium migration. ${ }^{5}$

\section{Mechanism of action of CGF on bone defects}

CGF, a new type of biological material contains a higher concentration of growth factors, fibrinogen, leukocytes, coagulation factors, CD34+ cells and VEGF (vascular endothelial growth factor).VEGF initiates vascular branch formation, induces vascular leakage and promotes endothelial cell proliferation. It also induces vascularization of the CGF membrane. EGF stimulates epidermal growth, keratinization, and promotes angiogenesis in the CGF membrane which causes gingival modifications. CGF enhances osteogenic differentiation and cell proliferation, prolongs the duration of growth factor activity, which is conductive for growth factor synergy. CD34+ cells induce neovascularization of the CGF membrane, ensuring the sealing of soft tissues. This enhances the effect of guided bone regeneration by promoting healing, improving wound stability required for attachment of a new connective tissue to the root surface. CGF is $3 \mathrm{D}$ - membrane network structure, which contains a high concentration of growth factors and platelets. These can be released and act over a prolonged period effectively. ${ }^{6,7}$

\section{Effect of CGF on osseointegration}

Osseointegration of dental implants is vital for stability, success and for a longer shelf life. (CGF) increases implant stability, accelerates osseointegration by increasing the differentiation of osteoblasts and healing of the bone around the implant.

CGF contains fibrinogen, growth factors, leukocytes, coagulation factors, endothelial growth factor, platelets for angiogenesis and tissue remodeling. It provides a matrix for cell migration. Platelets contain a high concentration of bioactive proteins required for healing, growth, and cell morphogenesis. CGF increases FGF- $\beta$ or VEGF release, which are essential for angiogenesis and enhancing neutrophil migration by performing integrin release.

Implant cavities are covered with CGF membrane before the implants are placed. The bone formation is increased in the CGFtreated implant site. CGF contains more growth factors than other platelet preparation thus has favorable effects on healing period of the implant. It accelerates the osseointegration of the implant and affected the stabilization values positively. ${ }^{8}$

\section{Conclusion}

Periodontal surgical procedures using growth factors are a new method that accelerates and enhances the process of natural wound healing and bone regeneration mechanism. Concentrated Growth Factor (CGF) is a biological repairing material, a new generation of blood extract, which stimulates and accelerates the bone formation and healing of tissues. CGF also improves the quality of the formed bone, enhances tissue regeneration, promotes stabilization of grafts and is effective in regulating inflammation. The success of this therapy lies in the local delivery of a high concentration of growth factors and proteins. This is similar to the physiologic wound healing, and supports reparative tissue process and local infiltration therapy, taking the surgical practice of regenerative techniques to a higher level.

\section{Acknowledgements}

None.

\section{Conflict of interest}

The author declares that there is no conflict of interest.

\section{References}

1. Jaishree Tukaram Kshirsagar, Rubine S. Innovation in regenerationConcentrated growth factor. International Journal of Applied Dental Sciences. 2017;3(2): 206-208.

2. Shuqi He, Mengjie Zhang, Zhaoqiang Zhang, et al. Application of CGF in Dental Clinic. HPBS. 2015;1(4):54-56. 
3. Bozkurt Dogan S, Ongoz Dede F, Balli U, et al. Concentrated growth factor in the treatment of adjacent multiple gingival recessions: a split-mouth randomized clinical trial. J Clin Periodontol. 2015;42(9):868-875.

4. Ramakrishnan T, Shobana P, Vidya Sekhar. Concentrated growth factor membrane - A novel barrier for accelerated repair of gingival defect along with sliding flap technique. Int J Cur Res Rev. 2016;8(21).

5. Gökmenoğlu C, Yavuz MC, Sadik E, et al. Treatment of Different Types of Bone Defects with Concentrated Growth Factor: Four Case Reports. Int $J$ Oral Dent Health. 2016;2(2).
6. Ying X, Chen Y, Luo N, et al. The Use of Concentrated Growth Factors and Autogenous Bone for Periodontal Soft Tissue Augmentation and Bone Regeneration: A Case Report. Electronic J Biol. 2017;13:3.

7. Jing Qiao, Jinyu Duan, Yong Zhang, et al. The effect of concentrated growth factors in the treatment of periodontal intrabony defects. Future Sci $O A$. (2016);2(4):FS136.

8. Cagasan Pirpir, Onur Yilmaz, Celal Candirli, et al. Evaluation of effectiveness of concentrated growth factor on osseointegration. Int $J$ Implant Dent. 2017;3(1):7. 